\title{
Correction to: Effects of Cognitive Behavioral Therapy for Insomnia on Subjective-Objective Sleep Discrepancy in Patients with Primary Insomnia: a Small-Scale Cohort Pilot Study
}

\author{
Kohei Nishikawa ${ }^{1,2} \cdot K^{2}$ ichi Kuriyama ${ }^{1,3} \cdot$ Takuya Yoshiike $^{3} \cdot$ Atsushi Yoshimura ${ }^{1}$ Masako Okawa ${ }^{4}$. \\ Hiroshi Kadotani ${ }^{5} \cdot$ Naoto Yamada $^{1,6}$
}

Published online: 20 August 2021

(c) International Society of Behavioral Medicine 2021

\section{Correction to: Cognitive Behavioral Therapy Rev https://doi.org/10.1007/s12529-021-09969-x}

This article was corrected to unblinded information in the Ethics Section.

Publisher's Note Springer Nature remains neutral with regard to jurisdictional claims in published maps and institutional affiliations.

The original article can be found online at https://doi.org/10.1007/ s12529-021-09969-x.

Kohei Nishikawa

shiga@cbtcenter.jp

1 Department of Psychiatry, Shiga University of Medical Science, Seta Tsukinowa-Cho, Otsu, Shiga 520-2192, Japan

2 Japan CBT Center, CG building F4, 3-12 Chuo-cho, Hikone, Shiga 522-0063, Japan

3 Department of Sleep-Wake Disorders, National Institute of Mental Health, National Center of Neurology and Psychiatry, 4-1-1 Ogawa-higashi, Tokyo, Kodaira 187-8553, Japan

4 Sleep Health Promotion Organization Institute of Neuropsychiatry, 4-45-16 Otsuka Bunkyo-ku, Tokyo 112-0012, Japan

5 Department of Sleep and Behavioral Sciences Center, Shiga University of Medical Science, Seta Tsukinowa-Cho, Otsu, Shiga 520-2192, Japan

6 Kamibayashi Memorial Hospital, 89-1 Okuchoorikuchinishi, Ichinomiya, Aichi 491-0201, Japan 